\title{
Cooling orbital frontal cortex disrupts matching-to-sample and visual discrimination learning in monkeys
}

\author{
MARY LOU VOYTKO \\ State University of New York Upstate Medical Center, Syracuse, New York
}

\begin{abstract}
Matching-to-sample and visual discrimination performance was examined during cooling of the orbital cortex in monkeys. Cooling produced severe deficits in simultaneous match-to-sample and delayed match-to-sample performance across all delays, 0-60 sec, and resulted in substantial difficulties in learning visual discriminations. Retention of discriminations learned during cooling of the orbital cortex was unaffected when tested under cooling or noncooling conditions. Discriminations learned prior to cooling were also not affected by orbital cooling. These results suggest that the orbital cortex is important in visual learning but not in retention, and are similar to findings on these tasks with anterior temporal lobe cooling.
\end{abstract}

Lesion studies have demonstrated that the temporal pole is engaged in visual processing necessary for the performance of delayed matching tasks and visual discriminations (Brown, Rosvold, \& Mishkin, 1963; Delacour, 1977; Harlow, Schiltz, Blomquist, \& Thompson, 1970; Jones \& Mishkin, 1972). In a recent series of experiments, we were able to disrupt performance on a visual delayed match-to-sample task by cooling the temporal pole (Horel \& Pytko, 1982; Horel, Voytko, \& Salsbury, 1984). In the latter study, we also demonstrated that although temporal pole cooling disrupts the ability of animals to learn visual discriminations, it does not severely affect their retention of such discriminations if they are learned sufficiently prior to cooling.

The temporal pole's major cortical associations outside the temporal lobe appear to be with the frontal lobe. Temporal pole cortex lesions result in fiber degeneration spreading over almost all of the orbital surface, as well as over the cortex ventral to the principal sulcus on the lateral surface of the frontal lobe (Jones \& Powell, 1970; Pandya \& Kuypers, 1969); the ventral cortex consists of areas 46 and 12 and is referred to as the inferior prefrontal convexity (Rosenkilde, 1979; Walker, 1940). These anatomical relationships are reciprocal (Pandya \& Kuypers, 1969; Van Hoesen, Pandya \& Butters, 1975).

The inferior prefrontal convexity has been the subject of a number of behavioral studies that have examined its involvement in visual tasks (e.g., Mishkin \& Manning, 1978; Passingham, 1975; Stamm, 1973). With a few exceptions, the orbital cortex, on the other hand, has been

This work was supported by Grant NS18291-01 from the National Institute of Neurological and Communicate Disorders and Stroke. The author wishes to thank James A. Horel for his advice and criticism throughout the project and is grateful to Kent Salsbury and Dorothy Joiner for their suggestions and comments in the construction of the cooling probes and the training of the animals. examined for its physiological (Ruch \& Shenkin, 1943; Stanley \& Jaynes, 1949) and emotional (Butter, McDonald, \& Snyder, 1969; Butter \& Snyder, 1972; Butter, Snyder, \& McDonald, 1970) functions more than for its mnemonic processes. It has been suggested that the orbital cortex is functionally part of a more extensive area that reaches into the temporal polar formation (Myers, 1967; Pribram \& Bagshaw, 1953; Pribram, Lennox, \& Dunsmore, 1950; Pribram, Mishkin, Rosvold, \& Kaplan, 1952). The present series of experiments examined the involvement of the orbital cortex in visual learning and retention, to see whether cooling the orbital cortex would produce deficits in tasks similar to deficits that have been seen with reversible cold lesions of the temporal pole (Horel et al., 1984).

Experiments 1-4 examined simultaneous match-tosample (SMS) and delayed match-to-sample (DMS) performance, over a series of delays ranging from 0-60 sec, with orbital cooling. Experiments 5 and 6 tested the ability of the animals to learn and retain object discriminations with the cold. The animals were also tested in these experiments for their ability to remember without a lesion a discrimination that was learned with a lesion. This can be done only with reversible functional lesions.

An anterior and posterior cooling probe were bilaterally implanted on the orbital cortex. This allowed testing of differences in match-to-sample performance while only one or the other of these probes was being cooled. Such bilateral single-probe cooling might indicate an areal difference within the orbital cortex, as well as differences in performance from simultaneous cooling of all probes, as we found in our earlier temporal lobe study (Horel et al., 1984).

The difficulty orbital animals have exhibited in various tests designed to assess problem-solving and instrumental functions has often been attributed to the lack of inhibition of perseverative tendencies (Brutkowski, 
Mishkin, \& Rosvold, 1963; Butter, 1969; McEnaney \& Butter, 1969). An analysis of perseverative errors was therefore made in the present study to determine whether perseverative interference was playing a significant role in disruption of delayed match-to-sample or visual discrimination performance.

Reversible cold lesions have been successfully used to uncover the functions of various temporal lobe (Fuster, Bauer, \& Jervey, 1981; Horel, 1984; Horel \& Pytko, 1982; Horel et al., 1984) frontal lobe (Bauer \& Fuster, 1976; Fuster, 1980; Fuster \& Bauer, 1974; Shacter \& Schuckman, 1967), and subcortical areas (Skinner \& Lindsley, 1967). Cold lesions appear to match the effects of removal of the same tissue (Alexander \& Goldman, 1977; Bauer \& Fuster, 1976), and there are many advantages to using cold lesions rather than surgical lesions. The functional lesions produced by cooling are reversible, thus allowing the animals to be used as their own controls. Behavioral effects can be measured before, during, and after cooling. The cold lesions are produced well after surgical trauma has dissipated, thereby decreasing the chance that recovery of function will obscure a valid behavioral effect of the lesion.

\section{METHOD}

\begin{abstract}
Subjects
Two experimentally naive Macaca fasicularis, one female weighing $3.0 \mathrm{~kg}$ (Animal 464) and one male weighing $4.7 \mathrm{~kg}$ (Animal 480), were used in these experiments. Measurement of their daily water intake was made several days before training. The animals were then water deprived and obtained all their water during the daily sessions in the testing apparatus. Daily weights were used to help adjust the amount of water received in the apparatus in order to maintain their health but ensure continuous performance. The animals were water deprived for the 5 days a week in which they were run. They were allowed free access to water at the end of the week for a $24-\mathrm{h}$ period. The water was then removed for $24 \mathrm{~h}$ in preparation for the next week of training.
\end{abstract}

\begin{abstract}
Apparatus
The training apparatus has been described elsewhere (Horel \& Pytko, 1982). Briefly, the animals were placed in a restraining chair so that they faced a horizontal array of three rear-projection screens mounted onto a black panel in a sound-attenuating room. The screens were $8.5 \times 5.5 \mathrm{~cm}$, separated by $5 \mathrm{~cm}$ between screens; they were hinged at the top and a press on a screen actuated a microswitch. Three carousel projectors presented stimuli on $35-\mathrm{mm}$ slides separately to the three screens. Rotary solenoids controlled shutters that separately interrupted the three projector beams. The projectors automatically advanced at the end of each trial. An orangeflavored drink, delivered to the mouth by a small tube, was used for reinforcement. The entire apparatus and the data collection were controlled by computer.
\end{abstract}

\section{Procedure}

The animals were preoperatively trained on a multiple-stimulus version of delayed match-to-sample (Mishkin \& Delacour, 1975; Overman \& Doty, 1980). The stimuli were 400 colored slides of common objects. A trial began with the appearance of an object, the "sample," on the center screen. Ten responses to the center screen produced a reward, extinguished the sample, and started a delay of either $0,15,30,45$, or $60 \mathrm{sec}$. The delay was randomly chosen from among the five durations, with the constraint that none was to be repeated until all had been presented once. At the end of the delay, a stimulus identical to the sample, the "match," appeared on one of the two side screens, and another object, the nonmatch, appeared on the other screen. The match and nonmatch randomly appeared on each of the side screens. The animals were required to respond twice to the match and received a reward for each response. The stimuli were extinguished following the second response to the matching stimulus and were followed by a $15-\mathrm{sec}$ intertrial interval (ITI). An incorrect response extinguished the stimuli and started a 35-sec ITI. Each stimulus appeared half the time as correct and half as incorrect and was systematically paired with different stimuli. The animals were run in 20 trial blocks for a total of 160 trials per day.

Cooling probes were implanted once the animals were performing at $90 \%$ correct or better at all delays. The animals were again placed in the apparatus 2 weeks postoperatively and were retrained to their preoperative performance levels. Once this level of performance was achieved, testing was begun.

Experiments 1 and 2: Delayed match-to-sample. The animals were run in alternating blocks of 10 trials with the cold on (experimental blocks) and 10 trials without the cold (control blocks) for a total of 100 trials per day for 2 days during DMS testing. This schedule allowed the testing of 20 trials at each of the five delays. Four-minute intervals were interposed when switching from a control block to an experimental block, and 7-11 min intervened when alternating from experimental to control blocks. These intervals allowed the behavioral effects of the cold or recovery from the cold to stabilize.

Experiments 3 and 4: Simultaneous match-to-sample. The animals were then trained and tested on an SMS task in the same apparatus. In the SMS task, the sample remained on while the match and nonmatch appeared, and the animal had only to compare the center screen with the two side screens. The simultaneous condition alternated randomly with a 30 -sec delay. The animals were trained to levels of $90 \%$ correct or better in both the SMS and 30sec-delay conditions before testing. Alternating blocks of 20 trials with the cold on and 20 trials with the cold off were used during testing. The time intervals between control and experimental blocks were the same as those used in DMS testing; these intervals allowed the behavioral effects of the cold or recovery from the cold to stabilize. The animals were tested for 80 trials per day for 2 days.

Experiments 5 and 6: Visual Discriminations. Learning and retention of visual discriminations were tested after DMS and SMS testing were completed. The apparatus was the same. The simultaneous visual discrimination began with a white light projected to the center screen. A response to the light extinguished it and exposed the discriminative stimuli on the two side screens. The animals received no reward for responding to the white light, but did receive a reward for each of the two required responses to the correct stimulus. A response to the incorrect stimulus produced no reward. A 15-sec ITI followed a correct response and a 35-sec ITI followed an incorrect response. The stimuli consisted of randomly paired colored slides of objects not used in the previous experiments. The correct stimulus alternated sides randomly. The animals were run in blocks of 20 trials, and criterion in all cases was $90 \%$ correct or better in two consecutive 20-trial blocks.

In Experiment 5, prior to testing. the animals were given four object discriminations to learn so that we could estimate how long it would normally take them to learn these discriminations. For this the animals were run only to criterion. During testing. the animals were trained on object discriminations while being cooled. If criterion was reached before 160 trials, the animals were allowed to complete the 160 trials. However, if the animals did not achieve criterion within 220 trials, testing was continued the following day under the same conditions. Retention of the discrimination was tested with the cold applied for 160 trials. $24 \mathrm{~h}$ after learning criterion was attained.

Retention of the discrimination was tested again $24 \mathrm{~h}$ later, this time without the cold. In the preliminary tests, it was found that the animals learn these object discriminations very quickly without 
the cold, often with only one or two errors, which makes it difficult to test retention; when retested, they might show almost no errors even though they might have no recall of the objects. For this reason, we used the reversal of the discrimination learned under cold to test for retention without the cold. If the animals' initial performance was below chance, this would indicate that at least some of the task learned with the cold was retained without the cold. Retention of the reversal discrimination was tested without the cold $24 \mathrm{~h}$ after criterion was attained and was tested again $96 \mathrm{~h}$ later with the cold applied. This testing sequence was repeated for four object discriminations and is shown in Table 1.

Experiment 6 did not involve reversal discriminations. The animals were trained on an object discrimination without the cold for 160 trials on the first test day. Retention without the cold was tested for an additional 160 trials $24 \mathrm{~h}$ later. Finally, retention with the cold was tested for 160 trials after a further $24 \mathrm{~h}$ delay.

\section{Surgery}

The animals were anesthetized with sodium pentobarbital, and a small craniotomy exposed the lateral convexity of the frontal lobe. With direct visual guidance, chronic cooling probes were bilaterally implanted so that the loops of the probes covered the dura overlying the orbital cortex. The probe cylinders were fastened to the skull with stainless steel screws and cranioplastic cement.

\section{Cooling Apparatus}

Details of the chronic cooling probe's construction and methods of cooling are described elsewhere (Salsbury \& Horel, 1983). The probes consisted of loops of 23-ga. stainless steel tubing, $0.64 \mathrm{~mm}$, shaped with a casting of a female $M$. fasicularis brain. The male's probes were shaped with female casting and then enlarged to accommodate his larger brain size. Two pairs of probes were implanted in each hemisphere. In the male, the anterior probes measured $3 \times$ $16 \mathrm{~mm}$ and the posterior probes measured $3 \times 14 \mathrm{~mm}$. The anterior and posterior probes in the female measured $3 \times 15 \mathrm{~mm}$. The anterior and posterior probes were separated by $3 \mathrm{~mm}$ in both animals.

The 3-mm separation between the loops of each probe and between the probes themselves was based on previous results. We have found (Horel, 1984), as have others (reviewed in Brooks, 1983), that there is a steep isothermic gradient around a cold source of the size used in this experiment. Using the same cooling tubes in the temporal lobe, we found that with the tube cooled to $0^{\circ} \mathrm{C}$, the tissue temperature around the probes varied according to the following function:

$$
\mathrm{t}=\mathrm{a}_{0}+\mathrm{a}_{1} \mathrm{e}^{\mathrm{a}_{2} \mathrm{~d}}
$$

where $t$ and $d$ are temperature and distance, $a_{0}=37.0, a_{1}=-32.7$, and $a_{2}=-0.435$ (Horel, 1984). Synaptic transmission is suppressed at $20^{\circ} \mathrm{C}$ (Jasper, Shacter, \& Montplaisir, 1970), which, according to this function, is $1.5 \mathrm{~mm}$ from the probe. With a 1.1 -mm-diam cooling probe, we found that suppression of the tissue, as determined by behavioral measures, spread for about $1.6 \mathrm{~mm}$ (Horel \& Pytko, 1982). Thus, the cooling loops used in the present experiment should suppress the function of the cortex within the loop and about $1.6 \mathrm{~mm}$ beyond its circumference. The two loops together, in their intended positions, should suppress the function of the majority of the orbital cortex.

A thermocouple was soldered to the base of the loops of each probe and held in place with a sheath of Teflon tubing. The tubes and thermocouple wires, soldered to connectors, were cemented into a stainless steel cylinder $(3 \mathrm{~mm})$. Plastic caps covered the cylinders.

Pumps were used to drive methanol through Teflon tubing into a bath of dry ice and methanol. The cooled methanol was then pumped through Teflon tubes which fit over the stainless steel tubing of the probes. The temperature of the probes was controlled by varying the rate of flow of the methanol. The probe temperature was set at $0^{\circ} \mathrm{C}$ during the experimental (cold) trials and was monitored by Bailey Instruments' BAT 9 and BAT 12 thermometers, which are accurate to $0.1^{\circ} \mathrm{C}$.

\section{Histology}

The deeply anesthetized animals were perfused through the descending aorta with a normal saline solution followed by $10 \%$ unbuffered formalin. The brains were removed, examined, and photographed.

\section{Anatomical Findings}

The probes produced clearly visible impressions on the orbital surface in Animal 480, whereas only slight indentations were found in Animal 464. In all cases, the cortex looked normal around the impressions produced by the probes. The behavioral deficits observed with cooling are not likely to be the result of permanent damage made by the probes. Behavioral effects occurred only when the cold was applied, and disappeared when it was removed.

The design of the probes, and their lateral approach to the orbital cortex, may have allowed some of the probe tubing to contact the orbital ridge near the inferior branch of the arcuate sulcus. No impressions were found in this area in either of the animals. If any contact did occur, it was minimal, and it is highly unlikely that it contributed to the results.

The intent was to place the probes within the posterior-to-middle orbital area so that the posterior loop of the posterior probe would lie just anterior to the lateral sulcus. The actual locations of the probes proved to be more anterior than intended (Figure 1). The probes implanted in the right hemispheres of both animals were located 3-5 mm more anterior than those in the left hemispheres. The posterior probes of Animal 480 were 6-9 mm more anterior than their intended location and 5-6 mm more anterior than the posterior probes in Animal 464. In Animal 464, the posterior probe in the right hemisphere was located $4 \mathrm{~mm}$ more anterior than intended, whereas that in the left hemisphere was correctly placed. There was only a $1-1.5-\mathrm{mm}$ difference between the animals in the placement of the anterior set of probes, with Animal 480 having the more anterior placement. The differences between the animals in probe placements may partly account for the differences between the animals in performance when only the anterior or only the posterior probes were cooled.

\section{RESULTS}

\section{Experiment 1: DMS, Cooling All Four Probes}

The animals appeared normal and performed the basic operational requirements of the task (i.e., hitting the screens, drinking the liquid reward from the feeder tube) during cooling as they had preoperatively.

Table 1

Sequence of Visual Discrimination Tests for Experiment 5

\begin{tabular}{lccccc}
\hline Day & 1 & 2 & 3 & 4 & 7 \\
\hline Condition & Cold & Cold & Warm & Warm & Cold \\
\hline Task & Training on & $\begin{array}{c}\text { Retention of } \\
\text { Problem A }\end{array}$ & $\begin{array}{c}\text { Training on } \\
\text { reversal of } \\
\text { Problem A }\end{array}$ & $\begin{array}{c}\text { Retention of } \\
\text { reversal of } \\
\text { Problem A }\end{array}$ & $\begin{array}{c}\text { Retention of } \\
\text { reversal of } \\
\text { Problem A }\end{array}$ \\
\hline
\end{tabular}


Cooling both anterior and posterior pairs of probes produced deficits on DMS across all delays (Figure 2). Although there were some fluctuations in performance at the various delays, in most instances the animals performed near chance. In Experiment 2, we investigated whether these large decrements in DMS performance were due to a differential involvement of the anterior and posterior placements of the probes.

\section{Experiment 2: DMS, Cooling Anterior or Posterior Probes}

Cooling only the anterior pair of probes for the most part resulted in a large drop in performance at all delays in both animals (Figure 3A). Applying cold to the posterior set of probes produced a weaker and less consistent effect (Figure 3B). There was little difference between Animal 480's performance on DMS whether only the anterior or only the posterior pair of probes was cooled. Cooling the anterior probes in Animal 464, however, resulted in a greater overall decrease in performance on the task than posterior-probe cooling.

The loss at all delays in the DMS task during cooling suggests a perceptual or attentional problem rather than a mnemonic deficit. In Experiment 3, an SMS task was used, in which the sample remains on during the match. If the animal could perform well on this, it would rule out the possibility that the deficit was perceptual or attentional.

\section{Experiment 3: SMS, Cooling All Four Probes}

Surprisingly, Animal 480 had difficulty transferring to the SMS condition and required 4 training days to attain the $90 \%$ correct level on this task. Animal 464, however, achieved this level on SMS within 1 training day. Both animals were performing at $90 \%$ correct or better in the 30 -sec-delay condition from the start.

The animals performed at $90 \%$ correct or better under control conditions (Figure 4). Cooling produced severe decrements in performance for both animals at both the 30 -sec-delay and simultaneous conditions. Thus, we cannot rule out the possibility that the difficulty these animals experienced on DMS when the orbital cortex was cooled represents an inability to differentiate the stimuli. In Ex-
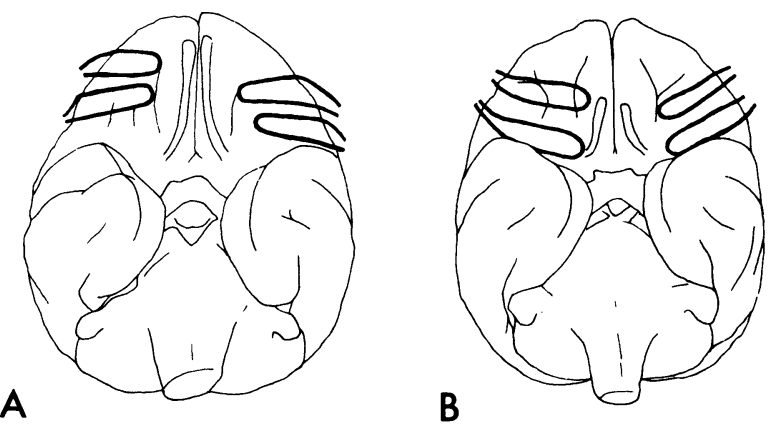

Figure 1. Ventral brain view of probe placements in Animal 480 (A) and in Animal 464 (B).

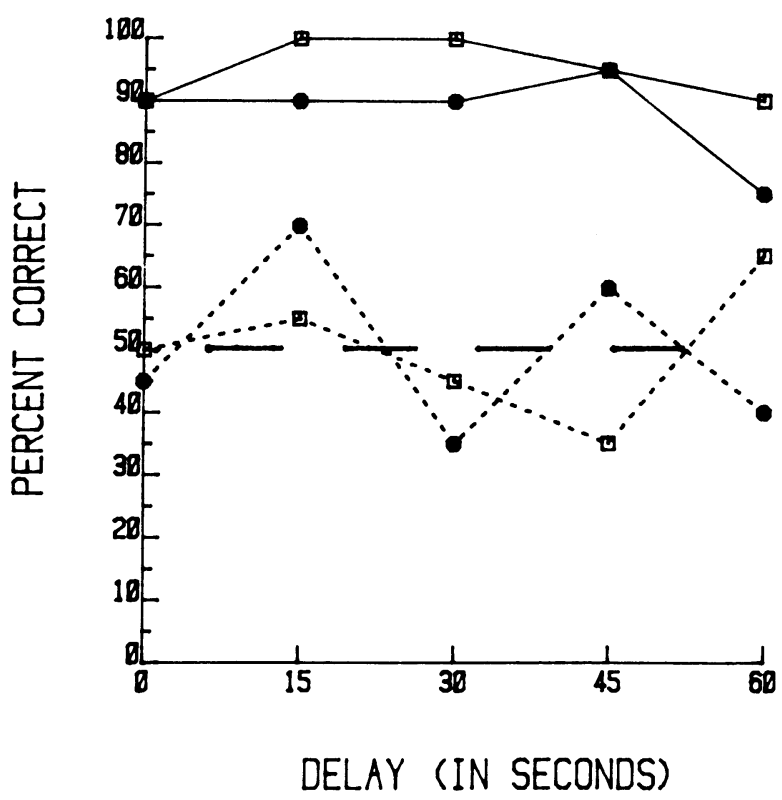

Figure 2. DMS performance during cooling of all four probes. Solid lines are control (warm) and dashed lines are experimental (cold) trials. Chance performance $(\mathbf{5 0 \%})$ is represented by a dashed horizontal line. $\bullet=$ Animal 480; $\square=$ Animal 464 .

periment 4 , we again examined the possibility of differential involvement of the anterior and posterior sets of probes.

\section{Experiment 4: SMS, Cooling Anterior or Posterior Probes}

Cooling the anterior probes produced decreases in performance in both the 30-sec-delay and SMS conditions in both animals (Figure 5A). Cooling the posterior probes of Animal 480 produced similar results in the SMS task and slightly less of a deficit in the 30-sec-delay condition than was found with anterior cooling (Figure 5B). Posterior-probe cooling in Animal 464 resulted in negligible decreases in performance in both conditions. These results are similar to those found with either anterior or posterior cooling in the DMS task: There was little difference between anterior and posterior cooling in Animal 480 , but a greater deficit with anterior than with posterior cooling in Animal 464.

The results of Experiments 3 and 4 suggest an impairment at the perceptual or input stage, rather than in processes of learning or memory. The following experiments examined the effects of cooling all four probes on the performance of visual discriminations and their reversals; we hoped to clarify the match-to-sample results obtained in Experiments 1-4.

\section{Experiment 5: Learning and Retention of Object Discriminations and Their Reversals}

The animals learned the object discriminations very rapidly under control conditions (Figure 6, WARM). In most cases learning occurred in less than 20 trials. Cool- 

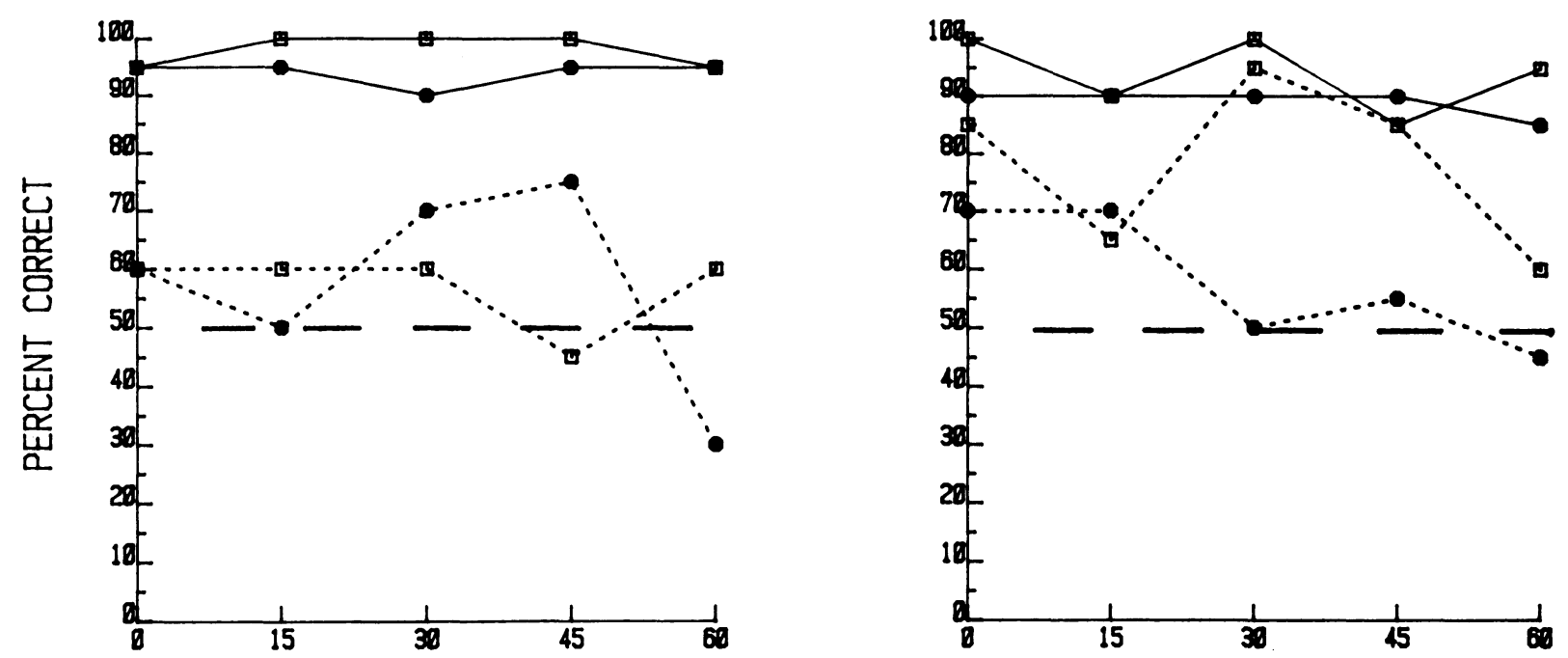

DELAY (IN SECONDS)

A

B

Figure 3. DMS performance during cooling of anterior probes (A) and posterior probes (B). See Figure 2 captions.

ing the orbital cortex, however, produced a severe retardation of learning (Figure 6, COLD). During these cooling sessions, the animals' behavior was an exaggeration of that displayed under control conditions. With cooling, the animals appeared confused and would frequently reach toward one stimulus, stop, and then respond to the opposite stimulus, whereas in control conditions this vicarious trial-and-error behavior was rarely seen. However,

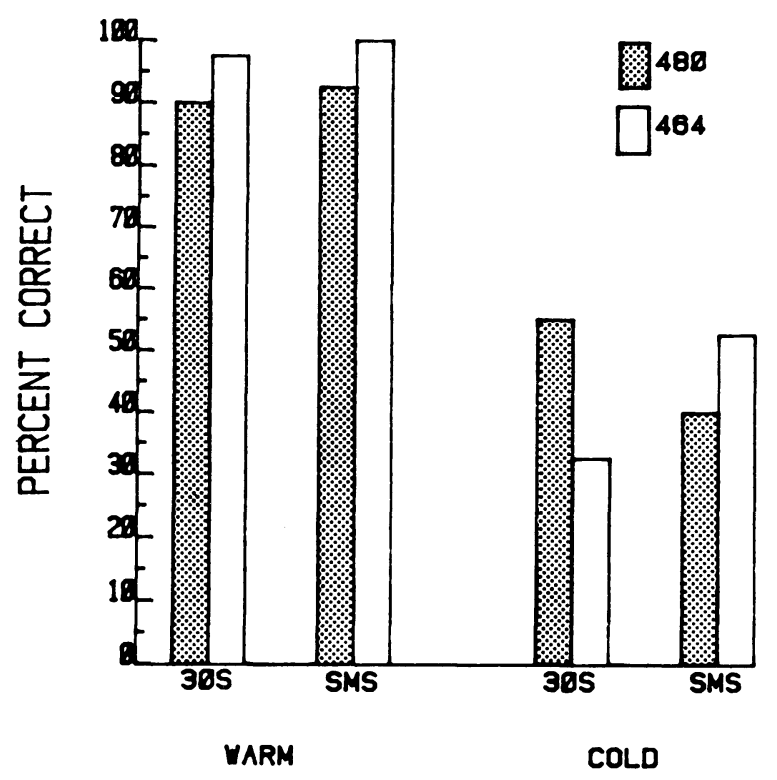

Figure 4. All four probes cooled with a 30-sec delay (30S) or a simultaneous match-to-sample (SMS). WARM = control trials; COLD $=$ experimental trials. the animals showed excellent retention of the discriminations when the cold was again applied $24 \mathrm{~h}$ after learning (Figure 7).

The animals were then tested without the cold for retention of the discriminations learned with the cold; this was done by examining their performance on the reversals of the discriminations. The animals had started well below chance on the first 20 trials of the reversal discriminations without the cold, but soon thereafter performed at high levels (Figure 8). A closer examination of the first 20 trials of each of the four reversal discriminations is presented in Figure 9. The animals responded to the incorrect stimulus $90 \%-100 \%$ of the time on the first 20 trials of the first discrimination, indicating excellent retention of the discriminations learned under the cold. On subsequent presentations, the development of reversal learning sets resulted in performance that approached chance levels; however, this performance was still considerably below that with the learning sets developed while learning discriminations without the cold.

The animals exhibited excellent retention of the reversals when tested $24 \mathrm{~h}$ later without the cold (Figure 10); however, when the cold was again applied $96 \mathrm{~h}$ later, the animals demonstrated poor retention of the same discriminations (Figure 11). This was surprising, because performance of the original discrimination when it was finally learned under the cold was excellent (compare Figures 7 and 11).

In summary, cooling of the orbital cortex produced severe learning deficits in object discriminations, but once learned, these discriminations were retained under both cooling and control conditions, as tested by the reversal 


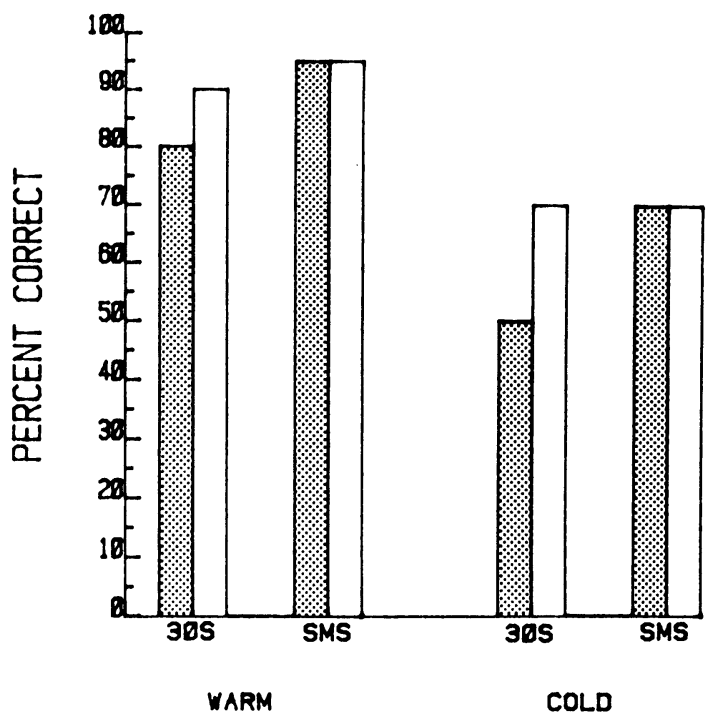

A

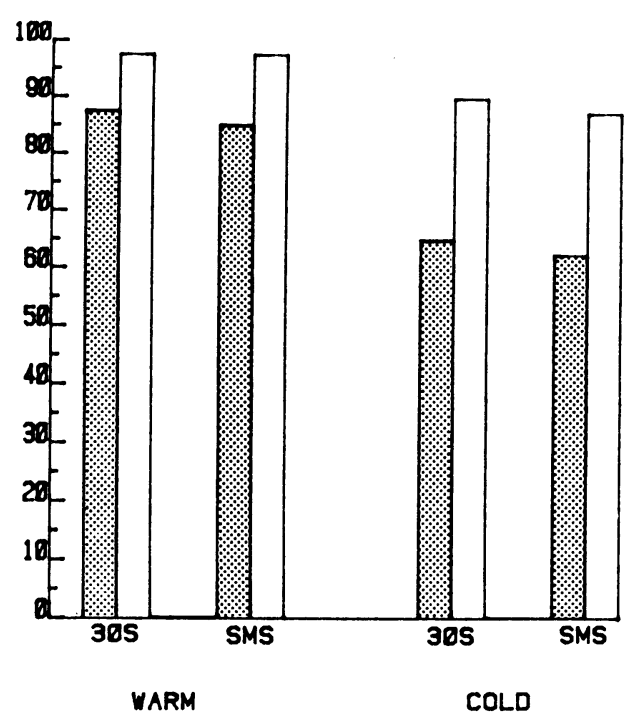

B

Figure 5. SMS and 30-sec performance during cooling of anterior probes (A) and posterior probes (B). See Figure 4 caption.

discriminations. In contrast, retention of the reversal discriminations that were learned under control conditions was found to be disrupted by cooling.

It is possible that the difference in retentive abilities that followed learning of visual discriminations with and without cooling may have been due to the use of reversals as the discriminations to be learned without the cold. Using the reversals of discriminations that had earlier been

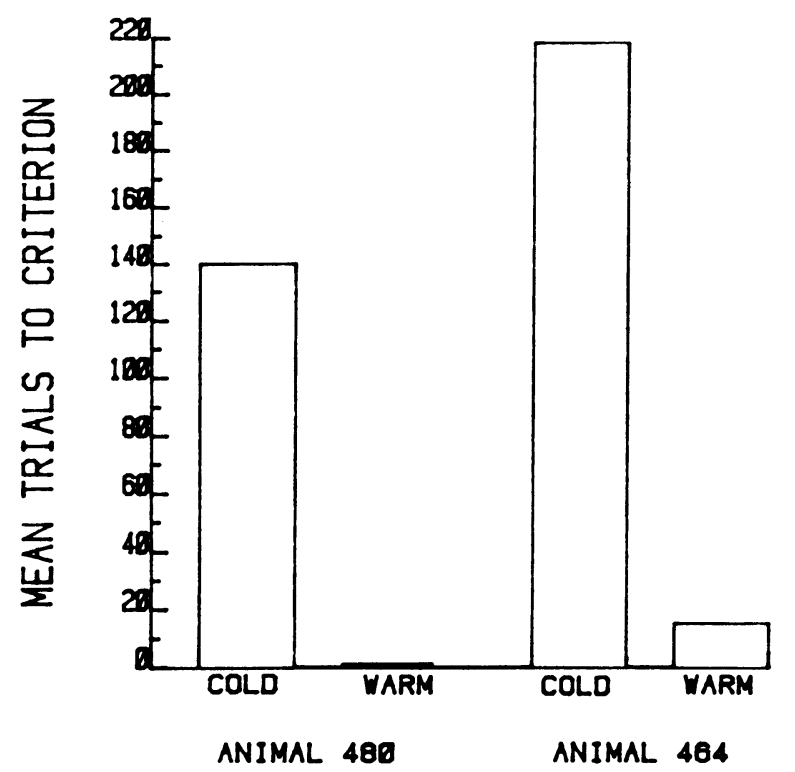

Figure 6. Learning object discriminations. WARM: Cold not applied. COLD: All four probes cooled. Data for each animal represent the mean of four discriminations. Animal 480 required 80-180 trials to reach criterion over the four discriminations during cooling; Animal 464 required $120-380$ trials. learned under cooling conditions may have caused some conflict within the animals because of interference between the original and the reversal discrimination when retention of the reversal was tested with the cold. Since there is no retention deficit with cooling, the animals could recall both the original discrimination and its reversal. However, because there is a learning deficit, the animals could not learn which of these two conditions was in effect when the retention of the reversal discrimination was examined with the cold. To test this hypothesis, the animals' ability to learn and retain object discriminations was further examined in Experiment 6.

\section{Experiment 6: Learning and Retention of Object Discriminations Without the Use of Reversal Discriminations}

The animals learned the object discrimination without the cold within 0-20 trials and showed good retention the following day (Figure 12). When the cold was applied $24 \mathrm{~h}$ later there was some initial difficulty in performing the discrimination; however, the animals consistently performed well above chance from the beginning and quickly reattained high levels of performance. When this retention performance with cooling is compared to the retention of the reversal discriminations with cooling in Experiment 5 (Figure 11), it suggests that the animals are able to retain discriminations under the cold that were learned originally without the cold. Thus, the poor retention of the reversal discriminations in Experiment 5 is probably due to the fact that both the original and the reversal are remembered, but because of a learning impairment, the animal has difficulty learning to choose which of those two opposite conditions is now correct when retention of the reversal discrimination is examined with the cold. 


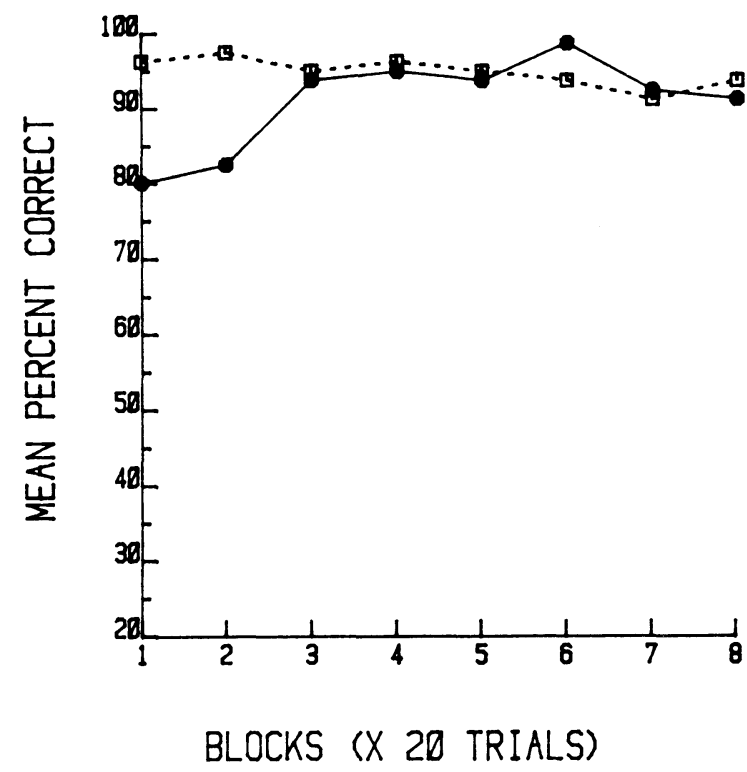

Figure 7. Mean retention of four object discriminations during cooling of all four probes. Solid line is Animal 480's performance. Dashed line is Animal 464's performance. Retention performance of Animal 480 ranged from $85 \%-95.6 \%$ correct over the four discriminations. Animal 464's performance ranged from $90 \%-97.5 \%$ correct.

\section{Perseverative Error Analysis}

An analysis was performed of errors made under control (warm) and experimental (cold) conditions for the DMS task of Experiment 1 and the learning of visual discriminations in Experiment 5. Perseverative error scoring followed that of Passingham (1975), who analyzed errors that occurred after a trial in which the animal had made a correct response. If the animal, in making an error, went to the same side as on the previous trial, the error was termed " parison of the percentage of perseverative errors made under control and cooling conditions in each task raises some doubts about the possibility that perseverative interference explains the orbital deficits (Table 2). In each task there was an increase in the total number of errors made and in the number of perseverative errors made under cooling conditions. However, the percentage of perseverative errors proved to be less with cooling than with noncooling for the DMS task, and to be similar between the two conditions in the learning of visual discriminations. Thus, it appears that perseverative interference can account for only about $50 \%$ of the errors made in DMS and visual discrimination performance during cooling of the orbital cortex. In most cases, this is less than or equal to what occurs under normal conditions.

\section{DISCUSSION}

Cold lesions of the orbital cortex produced behavioral deficits that were completely reversible, as indicated by the animals' high level of performance under noncooling (control) conditions. The behavioral effects occurred only when the cold was applied and disappeared when it was removed. These functional lesions demonstrated that the orbital cortex is important in delayed matching and visual discrimination performance.

Delayed match-to-sample performance was severely disrupted by cold lesions of the orbital cortex. The animals performed at chance levels across the series of delays from 0-60 sec, whether only one set or both sets of probes were cooled. Simultaneous match-to-sample performance was similarly affected. These results are consistent with a recent report by Mishkin and Bachevalier (1983), in which object recognition was examined following lesions of the ventromedial prefrontal cortex. The ventromedial lesions included the middle and medial orbital cortex as well as subcallosal and anterior cingulate cortex. The animals were unable to relearn a 10 -sec delayed nonmatch-tosample task following this lesion. It cannot be determined, without Mishkin's anatomical data, whether the cooling probes in the present study also functionally suppressed the orbital areas involved in their lesion. Nevertheless, the findings from both studies indicate that the orbital cortex is important in recognition memory.

Orbital frontal cooling also produced severe deficits in the learning of simultaneous object discriminations. In light of such debilitating learning deficits in paradigms involving repeated-trial learning, it is not surprising that we obtained devastating DMS deficits with cooling: each DMS trial is a visual discrimination which must be learned in that one trial. Few studies have examined the orbital cortex specifically for its possible involvement in visual

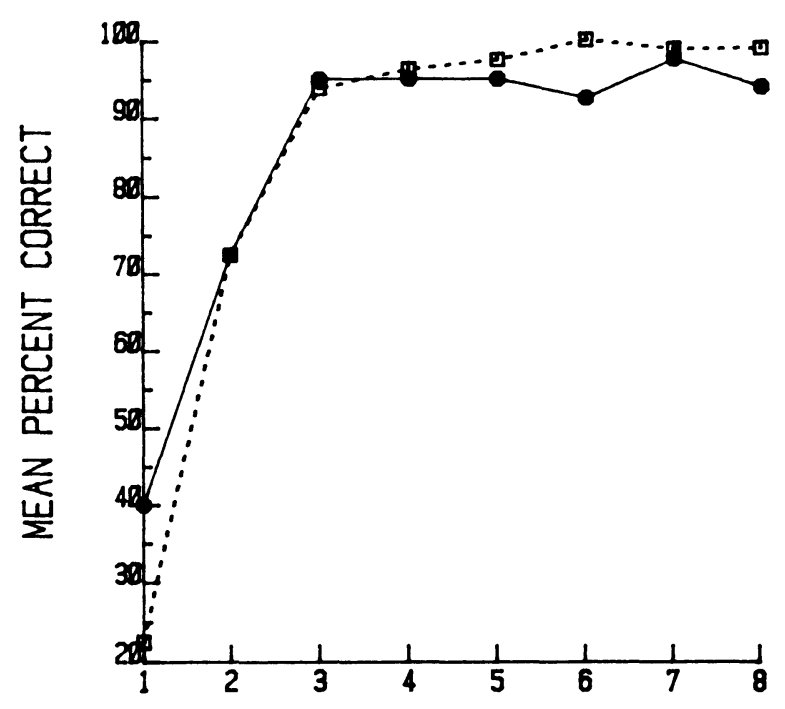

\section{BLOCKS (X 20 TRIALS)}

Figure 8. Learning without the cold the reversals of discriminations previously learned during cooling. Data represent the means of four reversal discriminations. Animal 480 required 20-80 trials to reach criterion over the four discriminations; Animal 464 required 20-40 trials to reach criterion. See Figure 7 caption. 


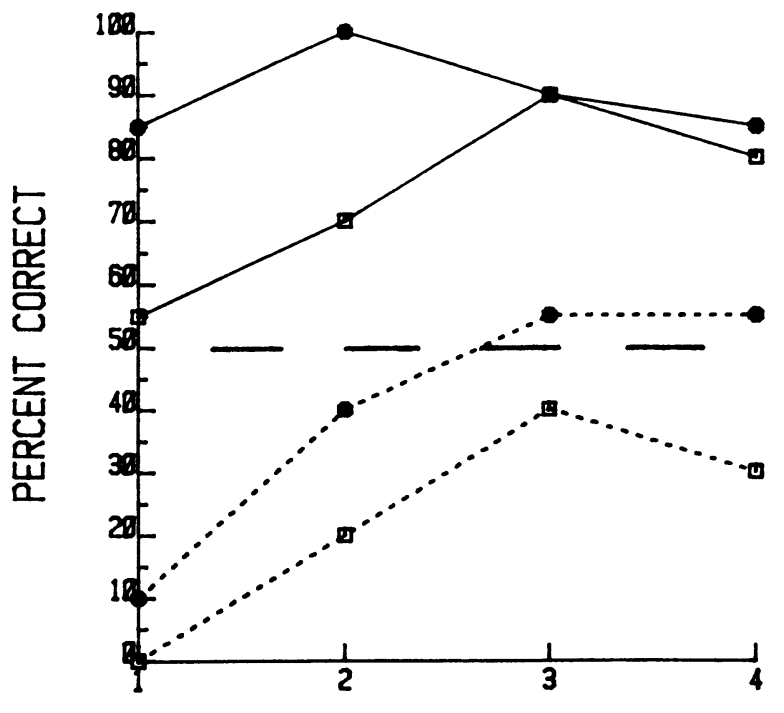

OBJECT PAIRS

Figure 9. Performance of the animals in the first 20 trials of each of the four reversal discriminations learned without the cold (dashed lines) and in the first 20 trials of the four discriminations learned under control conditions (solid lines). Chance performance $(50 \%)$ is represented by a dashed horizontal line. $\bullet=$ Animal 480; $\square=$ Animal 464.

learning. Mishkin (1964) tested orbital frontal animals for their ability to develop learning sets; however, the animals were tested for only 10 trials per problem and thus were never examined for their ability to learn visual discriminations to a high level of performance. Iversen and Mishkin (1970) reported that lesioned animals had no difficulties learning a simultaneous or successive visual discrimination; however, their orbital lesions were more restricted than those in the present experiment. Visualdiscrimination learning deficits have been reported following a combined lesion of the orbital and inferior prefrontal convexity (Goldman, Rosvold, \& Mishkin, 1970; Jones \& Mishkin, 1972; Passingham, 1972). Lesions of the inferior prefrontal convexity alone do not produce deficits in learning visual discriminations (Butters, Butter, Rosen, \& Stein, 1973; Iversen \& Mishkin, 1970; Oscar-Berman, 1978). Our cooling results indicate that the orbital cortex is important in learning visual discriminations, and therefore played a significant role in producing the learning deficits observed in previous studies. The particular learning mechanism that is suppressed by orbital cooling is not defined by our results.

The match-to-sample and visual-discrimination learning deficits could have resulted from the animals' experiencing some perceptual, sensory, motivational, or nonspecific problem, rather than learning difficulty. The retention findings of the visual-discrimination experiments of this study argue against these possibilities. The animals' retention of the discriminations proved to be affected very little by cooling of the orbital cortex. The animals ex- hibited good retention under both cooling and control conditions of the discriminations learned with the cold and were not severely affected by cooling when tested for retention of a discrimination learned under control conditions in Experiment 6. These retention results support the argument that the DMS or learning deficits were not due to perceptual or sensory problems, and indicate that the orbital cortex is not important in retention of simultaneous visual discriminations. During both learning and retention tests under cold, the animals continued to work at their normal pace, which suggests that the problem in learning was also not one of reinforcement or motivation.

Retention of visual discriminations following tissue removal of the orbital cortex has been the concern of two studies. Pribram et al. (1952) found no discrimination retention deficits with lesions of the middle and medial orbital cortex. Brutkowski et al. (1963), on the other hand, did find impairments in the retention of a preoperatively learned discrimination. Perhaps the reason for these different retention results is that different visual discrimination tasks were used in the two studies: simultaneous discriminations by Pribram et al., and successive go/no-go discriminations by Brutkowski et al. Successive discriminations are more difficult tasks to perform, and there appears to be a relationship between task difficulty and impairment following temporal and frontal lesions (Fuster, 1980; Gross, 1973; Pribram \& Mishkin, 1955). The present experiment examined simultaneous discrimination performance and obtained results that were con-

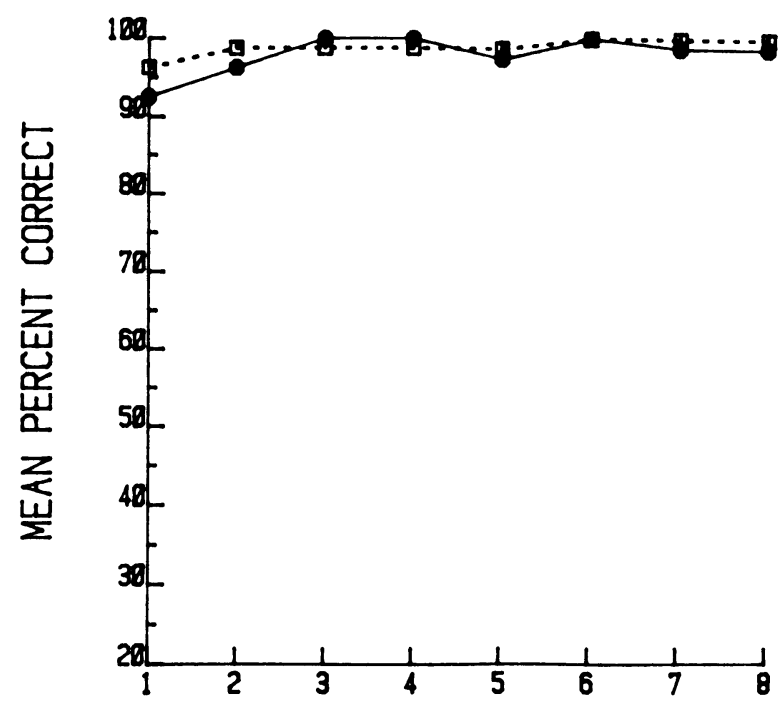

\section{BLOCKS (X 20 TRIALS)}

Figure 10. 24-h mean retention performance without the cold of four reversal discriminations. Animal 480's retention performance ranged from $96.8 \%-98.8 \%$ correct and Animal 464's performance ranged from $98.8 \%-99.4 \%$ correct over the four discriminations. See Figure 7 caption. 


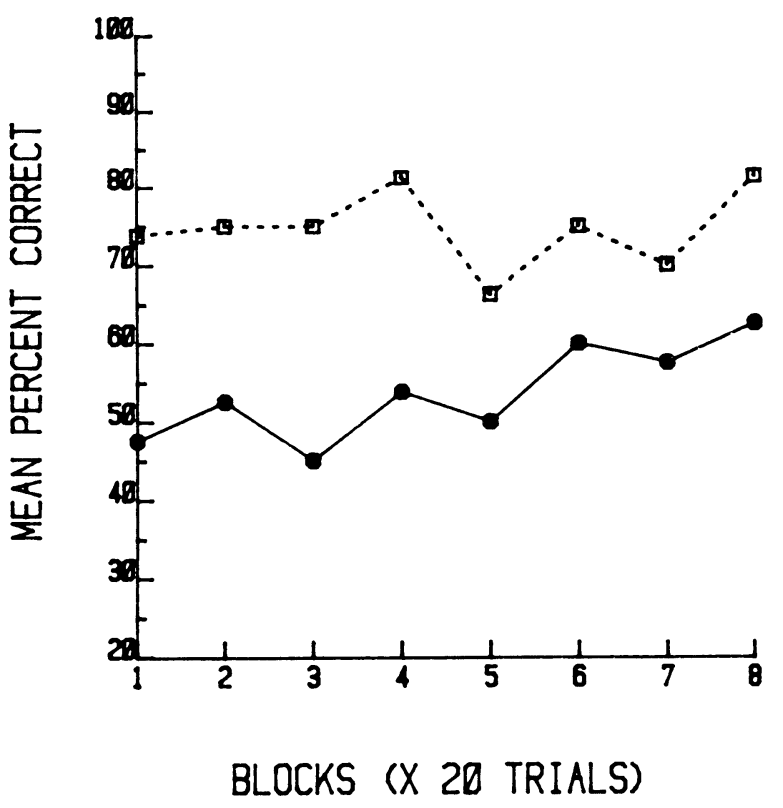

Figure 11. Ninety-six-hour mean retention performance of four reversal discriminations with cold applied. The range of retention performance over the four discriminations was $48.8 \%-59.4 \%$ for Animal 480 and $46.9 \%-95.6 \%$ for Animal 464. See Figure 7 caption.

sistent with the findings of Pribram et al., who also used simultaneous discriminations.

Poor performance was seen when retention of reversal discriminations was tested with the cold $96 \mathrm{~h}$ after reversal learning criterion had been achieved. The animals' retention with the cold of the reversal discrimination was at or near chance levels for most of the retention testing. If the animals were remembering only the original dis- crimination learned under the cold much earlier in the testing sequence, their performance levels should have been considerably below chance. Similarly, if they remembered only the reversal discrimination, their performance should have been considerably above chance levels. Neither of these outcomes was found with orbital cooling.

The animals' reversal performance scores imply that the animals may have remembered both the original and the reversal discriminations at the time that the retention of the reversal discriminations was being tested with the cold. The reversal-retention deficits may have occurred because of interference between these opposing discriminations. This interference, coupled with a severe inability to learn, could explain the reversal-retention scores we obtained during cooling of the orbital cortex. Some support for this interpretation was found in Experiment 6, which showed that the animals remembered with the cold a discrimination learned without the cold.

The term "perseverative interference" has commonly been invoked to explain the difficulties orbital frontal animals have in performing a variety of tasks. Reversal discriminations have been extensively used to demonstrate and examine the perseverative tendencies of such animals (Brutkowski et al., 1963; Iversen \& Mishkin, 1970; McEnaney \& Butter, 1969; Mishkin, 1964). In these tasks, the orbital frontal animals will continue responding to the previously correct stimulus beyond normal limits.

An analysis of perseverative errors made during DMS performance and visual discrimination learning shows that the percentage of perseverative errors made during cooling of the orbital cortex was less than or equal to the percentage of perseverative errors made under control conditions. This analysis indicates that there are other fac-

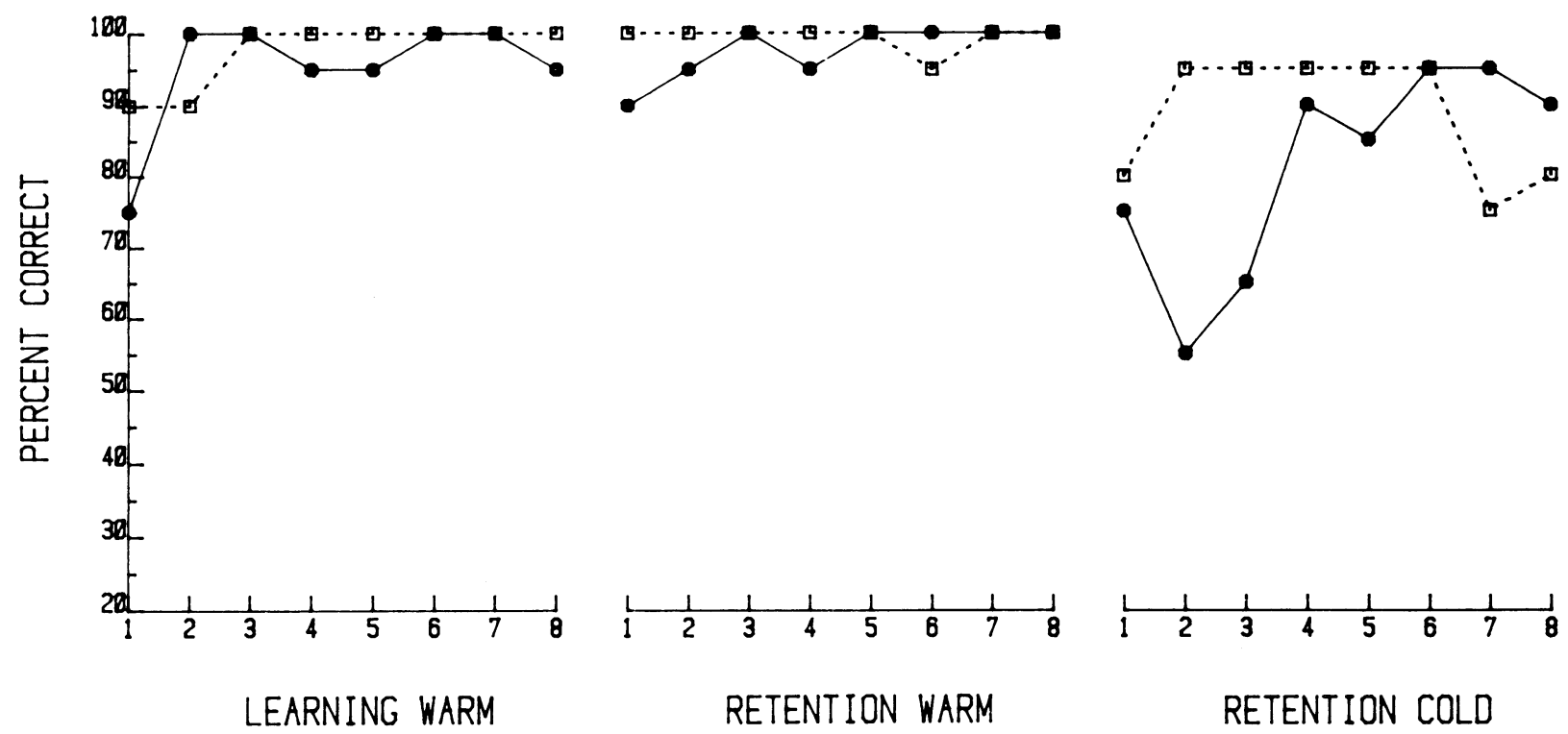

Figure 12. Learning and retention of an object discrimination without the cold and retention with all four probes cooled (RETENTION COLD). See Figure 7 caption. 
Table 2

Total Errors Made in DMS Across All Delays and in Learning Four Visual Discriminations, Under Control (Noncooling) and Experimental (Cooling) Conditions

\begin{tabular}{crcccc}
\hline & \multicolumn{2}{c}{ Control } & & \multicolumn{2}{c}{ Experimental } \\
\cline { 2 - 3 } \cline { 5 - 6 } Animal & DMS & Discriminations & & DMS & Discriminations \\
\hline 480 & $12(58.3 \%)$ & $30(53.3 \%)$ & & $50(50.0 \%)$ & $344(52.6 \%)$ \\
464 & $5(80.0 \%)$ & $9(44.4 \%)$ & & $52(46.0 \%)$ & $261(52.5 \%)$ \\
\hline
\end{tabular}

Note-Numbers in parentheses are the percentage of perseverative errors made during each task. Only those errors were analyzed which followed a trial on which the animal made the correct response.

tors contributing to the deficits following orbital lesions. The results of the present experiments suggest that a deficit in learning, but not in retention, is one of these factors. A learning deficit alone could account for both the DMS and visual discrimination performances observed during cooling of the orbital cortex. As mentioned previously, a learning deficit without a retention deficit would also explain the reversal-retention deficits obtained with cooling.

Cooling of the orbital cortex produced results that were similar to those found with cooling of the anterior temporal lobe. The delayed and simultaneous match-to-sample deficits produced by orbital cooling mirrored deficits found with cooling of the entire temporal pole (Horel et al., 1984). As is not the case with temporal pole cooling, however, we found no interaction between cooling and delays on the DMS task with individual cooling of the probes.

The visual-discrimination learning deficits produced with orbital cooling seem somewhat less severe than the learning impairments that were obtained with temporal pole cooling (Horel et al., 1984). The animals in Horel it al.'s study were unable to learn object discriminations rvithin 160 trials, although under control conditions they learned such discriminations within 0-20 trials. In a recent study (Voytko, in press), I found that although temporal pole cooling does severely retard learning of visual discriminations, the animals are eventually able to acquire them. Thus, cooling of either the temporal pole or the orbital frontal cortex results in deficits in learning visual discriminations, but does not prevent their eventually being learned. Similarly, retention of visual discriminations learned prior to cooling are not severely affected by cooling of either the orbital cortex or the temporal pole (Horel et al., 1984; Voytko, in press).

The similarity between the effects of cooling the orbital and the temporal polar cortex suggests that these two cortical regions may be associates in the learning process, and therefore possibly components of the same functional system. Recently, the amnesic syndrome has been interpreted in terms of a disconnection between the temporal and frontal lobes, due to neuropathology either in the medial thalamus or in the frontal or temporal lobes themselves (Warrington \& Weiskrantz, 1982; Weiskrantz, 1982). The results of the present experiments indicate that the orbital cortex, as well as the temporal pole, may be a critical component of this proposed frontal-temporal sys- tem. The deficits that are produced with lesions of the temporal pole or the orbital cortex are deficits in learning, but not in the retention of discriminations learned prior to the lesion. This resembles the deficits seen in amnesia.

\section{REFERENCES}

Alexander, G. E., \& Goldman, P. S. (1977). Functional development of the dorsolateral prefrontal cortex: An analysis utilizing reversible cryogenic depression. Brain Research, 143, 233-249.

Bauer, R. H., \& Fuster, J. M. (1976). Delayed-matching and delayedresponse deficit from cooling dorsolateral prefrontal cortex in monkeys. Journal of Comparative \& Physiological Psychologv, 90, 293-302.

BRooks, V. B. (1983). Study of brain function by local, reversible cooling. Review of Physiology \& Biochemical Pharmacology, 95, 1-109.

Brown, T. S., Rosvold, H. E., \& Mishkin, M. (1963). Olfactory discrimination after temporal lobe lesions in monkeys. Journal of Comparative \& Physiological Psychology, 56, 190-195.

Brutkowski, S., Mishinin, M., \& Rosvold, H. E. (1963). Positive inhibitory motor conditioned reflexes in monkeys after ablation of orbital or dorsolateral surface of the frontal cortex. In E. Gutman (Ed.), Second symposium on peripheral and central mechanisms of motor functions (pp. 133-141). Liblice, Czechoslovakia: Czechoslovakian Academy of Sciences.

Butter, C. M. (1969). Perseveration in extinction and in discrimination reversal tasks following selective frontal ablations in Macaca mulatta. Physiology \& Behavior, 4, 163-171.

Butter, C. M., McDonald, J. A., \& Snyder, D. R. (1969). Orality, preference behavior and reinforcement value of nonfood objects in monkeys with orbital frontal lesions. Science, 164, 1306-1307.

Butter, C. M. \& \& Syder, D. R. (1972). Alterations in aversive and aggressive behaviors following orbital frontal lesions in rhesus monkeys. Acta Neurobiologiae Experimentalis, 32, 525-565.

Butter, C. M., SNyder, D. R., \& McDonald, J. A. (1970). Effects of orbital frontal lesions on aversive and aggressive behaviors in rhesus monkey. Journal of Comparative \& Physiological Psychology, 72, 132-144.

Butters, N., Butter, C., Rosen, J., \& Stein, D. (1973). Behavioral effects of sequential and one-stage ablations of the orbital prefrontal cortex in the monkey. Experimental Neurology, 39. 201-214.

Delacour, J. (1977). Role of temporal lobe structures in visual shortterm memory using a new test. Neuropsychologia, 15, 681-684.

Fuster, J. M. (1980). The prefrontal cortex: Anatomy, physiology and neuropsychology of the frontal lobe. New York: Raven Press.

Fuster, J. M., \& Bauer, R. H. (1974). Visual short-term memory deficit from hypothermia of frontal cortex. Brain Research, 91. 393-400

Fuster, J., Bauer, R. H. \& \& Jervey, J. P. (1981). Effects of cooling inferotemporal cortex on performance of visual memory tasks. Experimental Neurology, 71, 398-409.

Goldman, P. S., Rosvold, H. E., \& Mishkin, M. (1970). Evidence for behavioral impairment following prefrontal lobectomy in the in- 
fant monkey. Journal of Comparative \& Physiological Psychology, 70, 454-463.

Gross, C. C. (1973). Visual functions of the inferotemporal cortex. In R. Jung (Ed.), Handbook of sensory physiology: Vol. VII/3. Central Processing of visual information (Part B, pp. 451-482). Berlin: Springer.

Harlow, H. F., Schiltz, K. A., Blomquist, A. J., \& Thompson, C. I. (1970). Effects of combined frontal and temporal lesions on learned behavior in rhesus monkeys. Proceedings of the National Academy of Sciences, 66, 577-582.

HoREL, J. A. (1984). Cold lesions in inferotemporal cortex produce reversible deficits in learning and retention of visual discriminations. Physiological Psychology, 12, 259-270.

Horel, J. A., \& PytKo, D. E. (1982). Behavioral effect of local cooling in temporal lobe of monkeys. Journal of Neurophysiology, 47, 11-22.

Horel, J. A., Voytko, M. L., \& Salsbury, K. G. (1984). Visual learning suppressed by cooling the temporal pole. Behavioral Neuroscience, 98, 310-324.

IVERSEN, S. D., \& MishKin, M. (1970). Perseverative interference in monkeys following selective lesions of the inferior prefrontal convexity. Experimental Brain Research, 11, 376-386.

Jasper, H. H., Shacter, D. G., \& Montplaisir, J. (1970). The effect of local cooling upon spontaneous and evoked electrical activity of cerebral cortex. Canadian Journal of Physiology \& Pharmacology, 48, 640-652.

JoNES, B., \& MishKin, M. (1972). Limbic lesions and the problem of stimulus-reinforcement associations. Experimental Neurology, 36, 362-377.

Jones, E. G., \& Powell, T. P. S. (1970). An anatomical study of converging sensory pathways within the cerebral cortex of the monkey. Brain, 93, 793-820.

McEnaney, K. W., \& Butter, C. M. (1969). Perseveration of responding and nonresponding in monkeys with orbital frontal ablations. Journal of Comparative \& Physiological Psychology, 68, 558-561.

Mish KIN, M. (1964). Perseveration of central sets after frontal lesions in monkeys. In J. M. Warren \& K. Akert (Eds.), The frontal granular cortex and behavior (pp. 219-241). New York: McGraw-Hill.

Mishín, M., \& Bachevalier, J. (1983). Object recognition impaired by ventromedial but not dorsolateral prefrontal cortical lesions in monkeys. Society for Neuroscience Abstracts, 9, 29.

Mishkin, M., \& Delacour, J. (1975). An analysis of short-term visual memory in the monkey. Journal of Experimental Psychology: Animal Behavior Processes, 1, 326-334.

Mishkin, M., \& Manning, F. J. (1978). Non-spatial memory after selective prefrontal lesions in monkeys. Brain Research, 143, 313-323.

MYERS, R. E. (1967). Cerebral connectionism and brain function. In C. H. Millikan \& F. L. Darley (Eds.), Brain mechanisms underlying speech and language (pp. 61-72). New York: Grune \& Stratton.

OsCAR-Berman, M. (1978). The effects of dorsolateral-frontal and ventrolateral-orbitofrontal lesions on nonspatial test performance. Neuropsychologia, 16, 259-267.

Overman, W. H., \& Doty, R. W. (1980). Prolonged visual memory in macaques and man. Neuroscience, 5, 1825-1831.

Pandya, D. N., \& KuYPers, H. G. J. M. (1969). Cortico-cortical connections in the rhesus monkey. Brain Research, 13, 13-36.
Passingham, R. E. (1972). Visual discrimination learning after selective prefrontal ablations in monkeys (Macaca mulatta). Neuropsychologia, 10, 27-39.

Passingham, R. (1975). Delayed matching after selective prefrontal lesions in monkeys (Macaca mulatta). Brain Research, 92, 89-102.

Pribram, K., \& Bagshaw, M. (1953). Further analysis of the temporal lobe syndrome utilizing fronto-temporal ablations. Journal of Comparative \& Physiological Psychology, 99, 347-375.

Pribram, K. H., Lennox, M. A., \& Dunsmore, R. H. (1950). Some connections of the orbito-fronto-temporal limbic and hippocampal areas of the Macaca mulatta. Journal of Neurophysiology, 13, 127-135.

Pribram, K., \& MishKIN, M. (1955). Simultaneous and successive visual discrimination by monkeys with inferotemporal lesions. Journal of Comparative \& Physiological Psychology, 48, 198-202.

Pribram, K. H., Mishkin, M., Rosvold, H. E., \& Kaplan, S. J. (1952). Effects on delayed-response performance of lesions of dorsolateral and ventromedial frontal cortex of baboons. Journal of Comparative \& Physiological Psychology, 45, 565-575.

ROSENKILDE, C. E. (1979). Functional heterogeneity of the prefrontal cortex in the monkey: A review. Behavioral \& Neural Biology, 25, 301-345.

Ruch, T. C., \& Shenkin, H. A. (1943). The relation of area 13 on orbital surface of frontal lobes to hyperactivity and hyperphagia in monkeys. Journal of Neurophysiology, 6, 349-360.

Salsbury, K. G., \& Horel, J. A. (1983). A cryogenic implant for producing reversible functional brain lesions. Behavioral Research Methods \& Instrumentation, 15, 433-436.

Shacter, D., \& Schuckman, H. (1967). Effect of localized cortical cooling on delayed-response performance in the monkey. Journal of Comparative \& Physiological Psychology, 63, 477-479.

SKINNER, J. E., \& LiNDSLEY, D. B. (1967). Electrophysiological and behavioral effects of blockade of the nonspecific thalamo-cortical system. Brain Research, 6, 95-118.

Sтамm, J. S. (1973). Functional dissociation between the inferior and arcuate segments of dorsolateral prefrontal cortex in the monkey. Neuropsychologia, 11, 181-190.

Stanley, W. C., \& Jaynes, J. (1949). The function of the frontal cortex. Psychological Review, 56, 18-32.

Van Hoesen, G. W., Pandya, D. N., \& Butters, N. (1975). Some connections of the entorhinal (area 28) and perirhinal (area 35) cortices of the rhesus monkey: II. Frontal lobe afferents. Brain Research, 95, 24-38.

VoYTKo, M. L. (in press). Visual learning and retention examined with reversible cold lesions of the anterior temporal lobe. Behavioral Brain Research.

WALKER, A. E. (1940). A cytoarchitectural study of the prefrontal area of the macaque monkey. Journal of Comparative Neurology, 73, 59-86.

Warrington, E. K., \& Weiskrantz, L. (1982). Amnesia: A disconnection syndrome? Neuropsychologia, 20, 233-248.

WeISKRANTZ, L. (1982). Comparative aspects of studies of amnesia. Philosophical Transactions of the Royal Society of London, B298, 97-109.

(Manuscript received April 18, 1985; revision accepted for publication November 15, 1985.) 